\title{
Tres epigramas griegos inéditos del humanista Juan de Verzosa: una aportación a la historia de la Anthologia Graeca
}

\author{
Eduardo del Pino ${ }^{1}$
}

Recibido: 31 de Agosto de 2020 / Aceptado: 20 de Octubre de 2020

Resumen. En la Collectio Vulcaniana de la Bibliotheek Universiteit Leiden (Vul. 103 I, f. $2^{\text {v }}$ ) se conservan tres epigramas griegos inéditos del humanista hispano-latino Juan de Verzosa. Fueron escritos en Lovaina con motivo de la muerte, en 1545, del profesor de griego Rutgerus Rescius. La aparición de los textos aporta más luz al periodo docente del joven Juan de Verzosa en la lengua griega, así como a la historia de la Anthologia Graeca.

Palabras clave: Poesía Griega, Humanismo, Juan de Verzosa, Anthologia Graeca, Epigrama.

\section{[en] Three unknown Greek epigrams by the Spanish humanist Juan de Verzosa: a note to the history of the Greek Anthology}

Abstract. There are three unedited Greek epigrams written by the Spanish humanist Juan de Verzosa in the Collectio Vulcaniana of the Bibliotheek Universiteit Leiden. He composed them in Louvain after the death of the Greek professor Rutgerus Rescius. This discovery enlightens Verzosa's docent stay in Louvain. It adds as well a note to the history of the Anthologia Graeca.

Keywords: Greek poetry, Humanism, Juan de Verzosa, Anthologia Graeca, Epigram.

Sumario. 1. Verzosa y Rutgerus Rescius: claves de la actividad docente de Verzosa en Lovaina. 1.1. Juan de Verzosa, docente de lengua griega en Lovaina. 1.2. Rutgerus Rescius, professor de la Universidad de Lovaina. 1.3. El arrepentimiento de Verzosa por haber abandonado la carrera docente. 2. Los tres epigramas griegos de Verzosa desconocidos hasta ahora. 2.1. El texto griego de los epigramas con su traducción al español. 2.2. El léxico de los epigramas y sus modelos de la Antigüedad. 2.3. Una aportación a la historia de la Anthologia Graeca, deducida de los epigramas de Verzosa. 2.4. Morfología, métrica y estilo de los epigramas. 3. Conclusiones.

Cómo citar: del Pino, E. (2021), Tres epigramas griegos inéditos del humanista Juan de Verzosa: una aportación a la historia de la Anthologia Graeca, en Cuadernos de Filología Clásica. Estudios griegos e indoeuropeos 31, 217-234.

1 Universidad de Cádiz

eduardo.delpino@gm.uca.es

Este trabajo se inserta en el Proyecto de Investigación del Plan Nacional de I+D PGC2018-094604-B-C31 (MCIU/AEI/FEDER, UE). He consultado para él con el profesor Tomás Silva, a quien agradezco sus sugerencias e indicaciones bibliográficas. Como se verá, me ha sido de especial utilidad su tesis doctoral (citada en la bibliografía), dirigida por los profesores Máximo Brioso Sánchez y José Guillermo Montes Cala. Doy las gracias también a los revisores anónimos de la revista. Las traducciones de los textos son del autor de este artículo, salvo indicación contraria. 


\section{Verzosa y Rutgerus Rescius: claves de la actividad docente de Verzosa en Lovaina}

\subsection{Juan de Verzosa docente de lengua griega en Lovaina}

En este trabajo se presentan al lector los textos griegos de tres epigramas funerarios, inéditos hasta ahora, escritos por el humanista hispano-latino Juan de Verzosa (Zaragoza 1522/23 - Roma 1574), que con sus obras latinas póstumas constituye una muestra de la singular recepción de Horacio y Tácito en la segunda mitad del siglo $\mathrm{XVI}^{2}$. Los tres epigramas están motivados por la muerte en 1545 de Rutgerus Rescius, profesor de griego en el Colegio Trilingüe de la Universidad de Lovaina. Aportan algunos datos que iluminan los años que pasó el joven español en Lovaina como docente de griego.

El joven aragonés destacó en el Studium de su ciudad natal, Zaragoza, y su padre lo mandó a París en 1539 para que completara sus estudios. Ya entonces comenzó a dar clases de lengua griega. La información con que se cuenta sobre esta docencia (tempranísima, puesto que se marchó con quince años) procede de la biografía de Verzosa con que Luis de Torres, sobrino del arzobispo de Monreal (Sicilia) del mismo nombre, encabezó la edición póstuma de los Epistolarum libri IIII del aragonés:

quinctum decimum annum agens, Lutetiam, tanquam ad elegantis doctrinae mercaturam patris iussu profectus, triennio in nobilissimo gymnasio Graecas litteras adolescens gloriose docuit, auditoribus uel mille subsellia complentibus. Excitato post bello Perpinianensi, cum omnes Hispani edicto publico ea urbe pellerentur, Belgas cogitauit atque iter aggressus multos habuit suae nationis comites et Louanii maiori etiam applausu quam Parisiis in Graeca lingua iunentutem erudiuit ${ }^{3}$.

La indicación nobilissimo gymnasio permite suponer que, aunque no diera clases propiamente en uno de los Colegios de la Academia parisina (el núcleo de la Sorbona), pudo darlas en alguna de las otras instituciones que preparaban a los alumnos universitarios o afianzaban su formación (muchas de ellas a título meramente particular $)^{4}$.

2 Las fuentes principales para su biografía de época moderna están en las ediciones, con traducción y estudio, del Annalium liber primus (Maestre 2002: XXIII-LI), un libro de historia sobre el reinado de Felipe II; de la Charina siue Amores (Pérez-Morillo 2002: XVII-XXI), conjunto de elegías amorosas; y de las Epistolae (del Pino 2006: XXVII-XLV), imitadoras de las de Horacio. Véase también una actualización en Pérez-Morillo (2009-2013) y del Pino (2016).

3 Vid. de Torres (1577: $\left[3^{\mathrm{v}}-4^{\mathrm{v}}\right]$ y en particular $\left.3^{\mathrm{v}}\right)$. Luis de Torres, el arzobispo de Monreal, fue natural de Málaga y residió en Roma desde 1540 con diversos cargos. Debió de ser en Roma donde fraguó una duradera amistad con Verzosa, hasta el punto de que este le dejase en testamento sus escritos. El obispo dio las epístolas en verso a su sobrino del mismo nombre para que las publicara en la imprenta de los Mayda en Palermo. Traducción: "cuando tenía quince años su padre lo mandó a París para ampliar sus estudios; y, a pesar de ser adolescente, enseñó con fama la lengua griega durante tres años en un conocido colegio, llenando las bancas hasta un millar de alumnos. Después, al surgir la guerra de Perpiñán, como todos los hispanos fueron por decreto expulsados de París, se dirigió a Bélgica, camino que tomaron muchos compatriotas, y en Lovaina, con mayor aplauso incluso que en París, enseñó a la juventud la lengua griega".

4 Sobre la datación exacta de la edad con la que viajó a París y su situación económica, véase en particular Maestre (2002: XXIV-XXv); del Pino (2006: XxxII-XXx, n. 17). Sobre la presencia de Verzosa en la Universidad he consultado con resultado negativo Brockliss (1976); Bibliothèque Nationale Française (ed.), Acta Rectoria Parisiensis Universitatis; Farge (2006). 
No consta el grado de estudios que alcanzó el español en la Universidad de París y es posible que no los terminara, puesto que, como todos los estudiantes españoles, fue expulsado de París a causa de la guerra hispano-francesa. Nada tiene de extraño que muchos de ellos se desplazaran a Lovaina para continuar sus estudios, habiendo resistido esta ciudad un asedio francés en aquel mismo mes de julio y estando consolidada su posición pro-imperial a partir del 2 de agosto.

No obstante, Verzosa solo aparece en los registros de la comunidad universitaria brabantina en $1545^{5}$. Como es bien sabido, el incendio de la Centrale Bibliotheke en la Gran Guerra dejó muy mermada la documentación histórica de la Universidad lovaniense, así que poco ha podido documentarse en sus registros, al menos hasta ahora, de la titulación y docencia del español ${ }^{6}$.

En todo caso, no hay que restar valor a la información de Luis de Torres (que da siempre datos ciertos), cuando dice que Verzosa dio clases de griego en Lovaina con más éxito incluso que en París. Hay que tener en cuenta aquí que en la Universidad de Lovaina y en sus Collegia o "Pedagogies", existían professores y legentes. Los primeros eran los titulares de los puestos docentes -y sus principales responsables y beneficiarios- mientras que los segundos desarrollaban una labor subsidiaria de preparación de los candidatos para los exámenes y de apoyo a los professores, una labor que a veces realizaban en su propia casa y otras en el Collegium. Pero atendamos por el momento al profesor fallecido en 1545, Rescius.

\subsection{Rutgerus Rescius, professor de la Universidad de Lovaina ${ }^{7}$}

Rescius (Rutger Resen, Maaseik, ca. 1497 - Lovaina, 2 octubre 1545) se graduó como bachiller en Artes en París en 1514. En el mismo año se trasladó a Alcmar para dar clases de griego, y al año siguiente a Lovaina, donde aparece matriculado el 4 de octubre para estudiar Leyes. A la misma vez, como una forma de contribuir a su sustento, trabajó en la imprenta de Dirk Martens (principalmente como corrector de griego), con quien compartió también alojamiento hasta 1518 .

Es importante recordar aquí que la relación de Rescius con Erasmo fue muy importante e incluso decisiva en su carrera. El flamenco tuvo contacto epistolar con Erasmo y lo conoció personalmente cuando este llegó a Lovaina en julio de 1517. Gracias a su recomendación, Rescius fue nombrado profesor de griego del Trilingüe el 1 de septiembre de 1518, puesto en el que permaneció hasta su muerte. No obstante, sus relaciones con la Universidad no fueron buenas. Ya en diciembre de 1519 se vio implicado en las disputas entre el profesor Wilhelm Nesen (que había criticado a los teólogos lovanienses) y el Rectorado de la Universidad, llegando a sufrir prisión por ello. Pudo volver a su puesto gracias a la intercesión de Erasmo.

5 Vid. Schillings (1961: 4.1, 235, n 130): Ioannes Berzosa Hispanus. Sobre la estancia lovaniense de Verzosa ha tratado del Pino (2003: 174, y 2017a: 340, n. 4).

6 He consultado el archivo de la Centrale Bibliotheke y del Rijksarchiev en la ciudad, sin fruto. Por otro lado, se sabe que muchos estudiantes de la Academia Brabantina no se matriculaban oficialmente. Sobre la importancia relativa de los registros de matriculados, véase Janssens (1992).

7 La fuente biográfica fundamental para Rescius es el propio epistolario de Erasmo. Una biografía relativamente amplia y reciente, con la bibliografía fundamental, puede verse en Erbe \& Deutscher (2003). Véase también (cito por orden cronológico) Nèvo (1856: 202-207); de Vocht (1927: 140, nº 1437; 1934: 186, n. 3; 1951-1955: ad indicem); Reusens (1977: 51). Los estudios más recientes sobre el Colegio Trilingüe están en Papy (2017a y 2017b). 
Luego surgió un problema mayor cuando Rescius tomó matrimonio en 1525 y dejó de residir en el Trilingüe (algo que era de obligado cumplimiento para los profesores, en la idea de que así estarían más disponibles para sus tareas y sus alumnos). La ausencia de Rescius levantó la oposición del centro y la reducción de sus emolumentos. Pero, una vez más, Erasmo intermedió ante el Rectorado hasta conseguir que Rescius continuara.

El prestigio del flamenco como helenista llegó a ser tal que Francisco I le ofreció en 1527 una cátedra de griego en el Colegio Real de París, a la que Rescius, aconsejado por Erasmo, renunció para mantenerse en Lovaina.

Por otro lado, en 1519, año en que Dirk Martens cerró su imprenta, Rescius decidió abrir otra junto con Jean Sturm (alumno suyo entonces, a quien daba alojamiento, y más adelante rector del Gymnasium protestante de Estrasburgo). Rescius publicó allí varias obras de la Antigüedad griega (como Homero, Platón y Jenofonte), y de los padres griegos de la Iglesia ${ }^{8}$, así como algunas publicaciones para aprender griego (que le aportarían, es de suponer, ingresos por parte de los mismos estudiantes) ${ }^{9}$. Uno de estos últimos libros fue un manual de prosodia griega, explicada en latín, escrito por Verzosa para sus alumnos: el De prosodiis liber absolutissimus, nunc primum in gratiam linguae Graecae studiosorum editus (1544). En el prefacio del manual (pp. [A IV $\left.\mathrm{IV}^{\mathrm{r}} \mathrm{A} \mathrm{IV}^{\mathrm{v}}\right]$ ) Verzosa llama a sus destinatarios auditores meos, alusión esta que (parecida a la de París: subsellia complentibus) confirma una docencia directa y real.

Teniendo esto en cuenta, toma cuerpo la hipótesis de que la enseñanza de Verzosa estuviera en relación con la del flamenco, que sí consta como professor de griego en la Universidad ${ }^{10}$. Esa publicación los beneficiaría mutuamente, pues Rescius aportaría sus alumnos del Collegium como lectores y publicaba el manual, en tanto que Verzosa escribiría el libro y daría las clases. Los mismos datos académicos de Rescius avalan la hipótesis de Verzosa como legens del professor flamenco, ya que este no vivía en el Trilingüe, regentaba al mismo tiempo una imprenta y su reducida dedicación a la enseñanza despertaba malestar en las autoridades del Rectorado.

Aun más, puede que no fuera casualidad la coincidencia en el mismo año de la muerte de Rescius en 1545 y la aparición de Verzosa en los registros de la Universidad. Si Verzosa había ayudado a Rescius en su docencia, era un sustituto apropiado para el curso 1545-1546, máxime si la muerte de Rescius fue inesperada.

\subsection{El arrepentimiento de Verzosa por haber abandonado la carrera docente}

Otro aspecto que refuerza la hipótesis de que Verzosa comenzó una carrera docente en la Universidad de Lovaina de la mano de Rescius, es precisamente su arrepentimiento por haberla abandonado, algo que parece entenderse si se repasa el resto de su vida y se leen sus Epistolas, que tienen algo de testamento vital.

Ocurrió que Verzosa abandonó Lovaina en 1546 (o muy a finales del año anterior), porque el cuerpo diplomático español le ofreció un empleo mucho mejor pagado. Se celebraba ese año de 1546 Dieta imperial en Ratisbona y Verzosa fue reclamado allí

8 Como ejemplos véase Hesiodus (1538); Hippocrates (1533); Homerus, Ulyssea (1535), Ilias [1532]; Plato (1529) y (1529a); Xenophon (1530), Symposion; Euripides (1537), Andromachē; Aristophanes (1537), Aves. Se interesó también por la patrística griega y por el propio texto griego de la Biblia: Evangelia (1541); Chrysostomus (1529).

$9 \quad$ Por ejemplo, Clenardus (1530); y el Alphabetum Graecum (1536).

10 Véase del Pino (2017a: 340, n. 4). 
como traductor, precisamente por su prestigio en el campo de los idiomas ${ }^{11}$. Resultó que el joven español tuvo muy buena acogida en ese ámbito (en particular en el del culto diplomático Diego Hurtado de Mendoza, cuya biblioteca fascinó al humanista). Por eso, en ese mismo año aceptó el nombramiento de secretario de Mendoza, por entonces embajador en Trento y luego en Roma, a la vez que gobernador de Siena. De esta forma, Verzosa abandonó definitivamente Lovaina y marchó con Don Diego a Italia. Luego, cuando Mendoza cayó en desgracia, fue secretario de Gonzalo Pérez en Londres y Bruselas (1554-1559); a continuación (de 1559 a 1562) asesoró al embajador español en Roma Francisco de Vargas; y, a partir de esa última fecha, sirvió a la Embajada Española en Roma como director de su Archivo.

Por tanto, la vida de Verzosa se consumió sobre todo en servir como secretario de confianza a diversos diplomáticos. Por su conocimiento de idiomas (y por la experiencia que fue acumulando junto a los próceres de las cortes española y pontificia), fue también uno de los útiles 'agentes' o auténticos espías que Felipe II tenía repartidos por los territorios a los que alcanzaba su corona. Pero a cambio de esto, Verzosa tuvo que mudar de residencia según se le requería y pasó temporadas con escasos ingresos e incierto futuro.

Si se leen sus Epistolas latinas, se comprueba su disgusto con la vacuidad de la vida diplomática o política; el anhelo, en cambio, del tiempo dedicado al estudio y el deseo de regresar para ello a su patria. En definitiva, se constata el arrepentimiento de haber dedicado su vida a una tarea que acabó considerando de ingrata servidumbre.

Se puede citar al respecto el siguiente fragmento de sus Epístolas, donde se intuye su dolor por haberse marchado de Lovaina y haber sacrificado su prometedora carrera docente, a cambio del brillo pasajero de la corte (epist. 1, 38, versos 17-25):

Quin ego, ni sic deceptum uel uana cupido

Vel monitis abduxisset uagus error ab illis,

Quae bonus et felix genius suggesserat olim,

Pendulus a me uno, non ullo ab Rege, quotannis

Prolixam sentirem a me sortemque benignam.

Ast ubi dispexi quid res magis auctet Iberas

Vel firmet, placuique mihi placuique peritis,

Protinus illa omnis tumido laus languet, uti flos,

Cui fructum nebulae non concessere malignae ${ }^{12}$.

\section{Los tres epigramas griegos de Verzosa desconocidos hasta ahora}

Pues bien, de la presencia de Verzosa en Lovaina y de su relación con Rutgerus Rescius nos ha quedado otra muestra, inédita hasta ahora: los tres epigramas del

11 Es muy posible que la idea de incorporar al joven español a la Dieta partiera de conocidos suyos de su misma tierra aragonesa, quizás de Gonzalo Pérez, con el que nuestro autor tuvo una relación privilegiada en adelante.

12 Véase del Pino (2006: 346-347): “Es más, si yo, así de engañado por una vana ambición o por mi errante extravío, no me hubiese apartado de aquellos consejos que mi buen y feliz genio me había sugerido tiempo atrás, dependería de mí solo, no de ningún Rey, y podría sentirme dueño cada año de la prosperidad y bondad de mi suerte. Pero, después de que me hice entendido en cómo aumentar y consolidar los intereses políticos de Iberia, y que esto me satisfizo y yo satisfice a los entendidos, toda aquella gloria de este orgulloso languideció de inmediato, como la flor a la que nieblas malignas no concedieron el fruto". 
aragonés que se encuentran en la Collectio Vulcaniana de la Universidad de Leiden (ms. Vul. 103 I, f. 2 ${ }^{\mathrm{v}}$ ).

Resulta un tanto llamativo que estos epigramas se hayan conservado entre los papeles de Bonaventura Vulcanius (Brujas 1538 - Leiden 1614), profesor en Leiden desde 1581 hasta su muerte. Puede entenderse en el marco de los años de joven estudiante del bruguense, de los que pasó dos (1555-1557) en Lovaina, aunque diez años después que Verzosa ${ }^{13}$. Por las razones que sean, los epigramas de Verzosa debieron de conservarse en el ámbito de la Universidad Brabantina, quizás entre los estudiosos de griego o antiguos compañeros de Rescius; y, al residir allí Vulcanius, llegó a conocerlos y a guardar una copia. Cuando el conocido profesor de Leiden dejó todos sus papeles a su Universidad, quedaron entre ellos los epigramas de Verzosa ${ }^{14}$. El legado manuscrito de Vulcanius fue clasificado, tras su muerte, con cierto desorden. En los actuales legajos Vul. 97 y 103 se conserva su poemario latino y griego, junto con poesías dedicadas a él y algunas de otros autores ${ }^{15}$.

\subsection{El texto griego de los epigramas con su traducción al español}

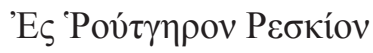

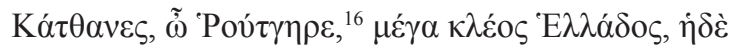

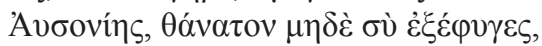

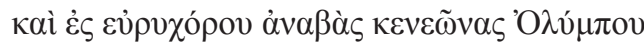

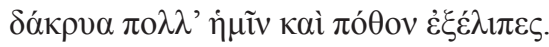

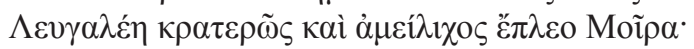

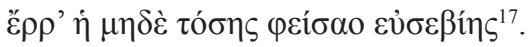

A Rutgerus Rescius

Falleciste, ;oh, Rutgerus! gran gloria de la Hélade y de la Ausonia, ${ }^{18}$ y tampoco tú te libraste de la muerte;

$\mathrm{y}$, ascendiendo a las extensas regiones del Olimpo ${ }^{19}$, nos dejas muchas lágrimas y dolor.

Moira, has sido dura y amarga en exceso, ¡vete!, ya que no has respetado su mucha piedad.

13 También pudo Vulcanius dar con esos epigramas más tarde, como veremos, sin que haya documentación en favor de una hipótesis u otra.

14 No hay ningún otro rastro de relación entre Vulcanius y Verzosa. Sobre el primero puede verse: de Vries de Heekelingen (1923), Dewitte (1983), Cazes (2010), Tournoy (2011a y 2011b), Silvano (2014), del Pino (2017b), Stefani (2019), García Pinilla (2020).

15 Los papeles son, en su inmensa mayoría, de mano de Vulcanius. Abarcan la producción de su vida, porque hay piezas que se sitúan en su estancia en España y una gran variedad posterior: sus arengas a los 'belgas' contra los españoles, su servicio al Príncipe Guillermo y sus años de docencia en Leiden. Sobre el contenido y el desorden de estos legajos véase van Dam (2010: 54-56). Sobre los poemas escritos en España véase García Pinilla (2020).

16 Ká $\tau \theta \alpha v \varepsilon \varsigma, \tilde{\omega}$ Pov́ $\gamma \eta \rho \varepsilon]$ cf. A.P. 7.576,1.

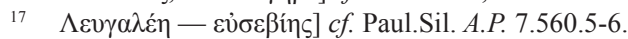

18 Nombre de Italia para los griegos. Verzosa destaca en Rescius su saber tanto en la lengua griega como en la latina.

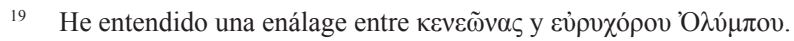


II

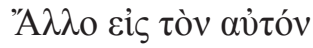

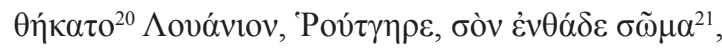

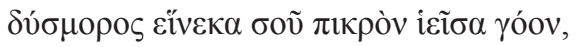

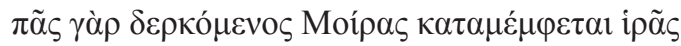

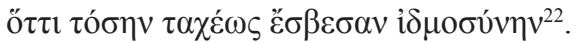

Otro para el mismo

Lovaina, Rutgero, acoge tu cuerpo mortal:

yo, triste, he vertido por ti un llanto amargo,

pues cualquiera que lo piense bien reprochará a las sagradas Moiras

que hayan apagado antes de tiempo tan gran sabiduría.

III

'A $\lambda \lambda \mathrm{o}$

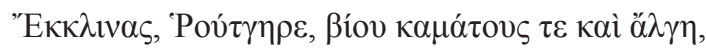

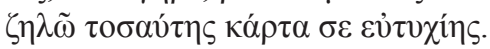

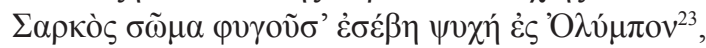

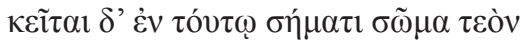

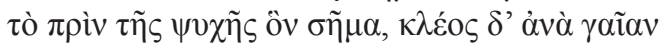

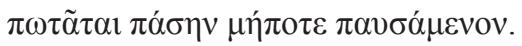

Otro

Te has librado, Rutgero, de los sufrimientos y dolores de la vida;

yo bien que te envidio por tanta suerte.

Tu alma, dejando tu cuerpo de carne, ha subido al Olimpo, y en esta tumba se queda tu cuerpo,

el que fue antes cárcel de tu alma; pero tu fama

volará por todas las tierras sin olvidarse nunca.

\subsection{El léxico de los epigramas y sus modelos de la Antigüedad}

Un breve estudio del léxico utilizado por Verzosa nos descubre que tomó por modelo para tejer sus epigramas varios epicedios de la Antigüedad, presentes en el libro séptimo de la Anthologia Graeca $^{24}$.

$20 \quad \theta \dot{\kappa} \alpha \tau$ ] cf. A.P. 7.3.1; 7.689.1.

$\left.{ }^{21} \theta \eta \dot{\kappa} \alpha \tau о-\dot{\varepsilon} \vee \theta \alpha ́ \delta \varepsilon \sigma \tilde{\omega} \mu \alpha\right]$ cf. Pilipp. Thesal. A.P. 7.362.1.

$22 \pi \tilde{\alpha} \varsigma-i \delta \mu o \sigma u ́ v \eta v] c f$. Agath. A.P. 7.602.9-10.

$\left.23 \psi v \chi \eta \dot{~ \varepsilon ̇} \zeta^{\prime} \mathrm{O} \lambda \hat{\mu} \mu \pi \mathrm{ov}\right]$ cf. Pilipp. Thesal. A.P. 7.362.3.

24 Una introducción a la composición poética en griego durante el Renacimiento y al estudio de sus fuentes léxicas hay en Montes Cala (1993). En cuanto a la Anthologia Graeca, como se sabe, la colección se basa en varias compilaciones sucesivas en el tiempo que acabaron en dos colecciones, la de Planudes (s. XIV) y la Palatina. Estas dos tienen una amplia base en común pero con piezas exclusivas en ambos casos. El manuscrito de Planudes se conservó en la Biblioteca Marciana de Venecia (ms. gr. 481) y a partir de él se hizo la primera impresión de la Anthologia por Láscaris, en la casa florentina de Álopa (1494). La segunda edición fue veneciana, por Aldo en 
El primer modelo es un epigrama de Paulo Silenciario. Acudamos a nuestros versos I 5-6 ${ }^{25}$, con los que Verzosa finaliza el primer epigrama:

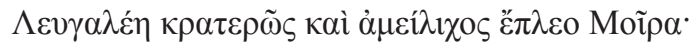

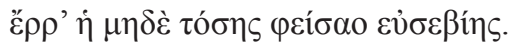

Podemos ver que ha redactado su final a partir del epigrama A.P. 7.560 de Paulo Silenciario por la muerte de un cierto Leoncio ${ }^{26}$ :

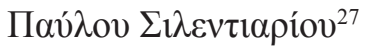

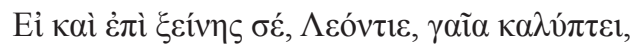

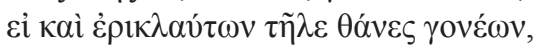

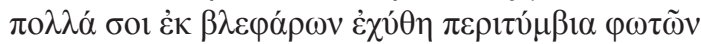

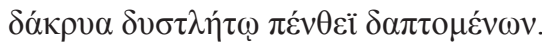

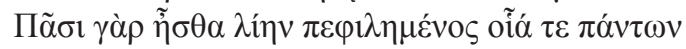

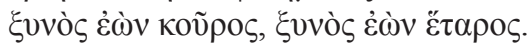

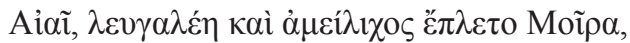

$\mu \eta \delta \dot{\varepsilon} \tau \varepsilon \tilde{\eta} \varsigma \eta \tilde{\eta} \beta \eta \varsigma, \delta \dot{\sigma} \sigma \mu \rho \rho \varepsilon, \varphi \varepsilon 1 \sigma \alpha \mu \varepsilon \dot{\varepsilon} \eta^{28}$.

En la comparación de Verzosa con su modelo, salta a la vista que Paulo Silenciario se dirige a la Moira en tercera persona ( $\left.\varepsilon^{\prime} \pi \lambda \varepsilon \tau 0\right)$, mientras que el joven humanista lo hace en segunda ( $\check{\varepsilon} \pi \lambda \varepsilon 0)$. Dejemos esta diferencia para comentarla en adelante.

Tratemos ahora de Verzosa II 3-4: el final de su segundo epigrama se construye a partir del de otro de Agatías el Escolástico, esta vez supuestamente para el joven Eustacio, sobrino del emperador ${ }^{29}$. Fijémonos en los versos de Verzosa II 3-4:

1531. El resto de ediciones de la Anthologia Graeca en el XVI se basaron en ella. La colección llamada Palatina fue descubierta para la "Res publica litterarum" por Claude Saumaise en 1606, en la Biblioteca del Palatinado en Heidelberg. Para las ediciones modernas de la Anthologia, puede verse Jacobs (1794-1814) y (1813-1817), obra esta última a partir de la cual decayó la publicación independiente de la Anthologia Planudea. Véase también (los cito por orden cronológico): Dübner (1871-1890), con traducción al latín, que publicó epigramas de inscripciones y de otros orígenes literarios (tercer volumen a cargo de E. Congruy); Stadtmüler, vol. 1 (1894), vol. 2 (1899), vol. 3 (1906); Paton (1979-1983) (=1916-1918): vol. 1 (1980=1916), vol. 2 (1970=1917), vol. 3 (1983-1917), vol. 4 (1979-1918); vol. 5 (1979-1918); Waltz (1960); Gow \& Page (1968). Reproduciré en adelante los epigramas de la Anthologia por la edición crítica de Pierre Waltz en la Collection Budé de Les Belles Lettres, teniendo a la vista también los aparatos críticos de Dübner y Stadtmüller. Citaré también el texto de Paton, sin aparato crítico como se sabe, pero que aporta traducción inglesa. En las notas que siguen (tomando pie en la edición de Waltz), la sigla $l$ se refiere al lemmatista que añadió resúmenes temáticos al manuscrito palatino, mientras que $c$ se refiere a una de las manos (la 'correctora') que intervino en él; la sigla Plan. se refiere al manuscrito de Planudes (Marcianus 486 de Venecia); cf. Waltz 1960: 1, xc.

25 Epigrama primero, versos 5-6, y así en adelante.

26 Sigo a Waltz (1960: 5, 93). Está también en Lascaris (1494: 300-301, 305-306); Dübner (1871-1890: I, 381, 486); Stadtmüller (1899: 387-388); Paton (1970: 300-301). Las noticias sobre los destinatarios están añadidas por $l$ (un lematista que añadió escolios al manuscrito conservado en Heidelberg con la sigla $P$ 23).

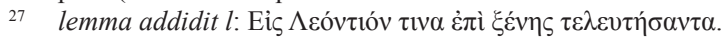

28 Traducción de los dos últimos versos: “¡Ay!, te alcanzó una Moira dura y amarga, y que no respetó, desgraciada, tu juventud!".

29 Según el lema que abajo cito, este Eustacio, muerto con quince años, sería hijo de otro Eustacio "el grande, antiguo prefecto". Podría tratarse (dice Waltz 1960: 5, 109) de un Eustacio que fue prefecto del pretorio en los años 505-506, bajo el emperador Anastasio I, según señala Dübner (1871-1890: 1, 480). 


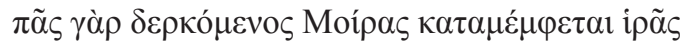

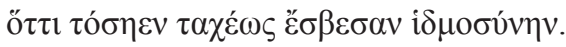

El epigrama de Agatías el Escolástico es A.P. 7.602 ${ }^{30}$ :

A $\gamma \alpha \theta i ́ o v ~ \Sigma \chi 0 \lambda \alpha \sigma \tau 1 \kappa o \tilde{v}^{31}$

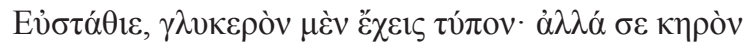

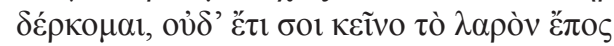

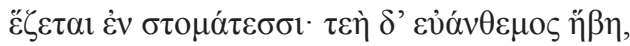

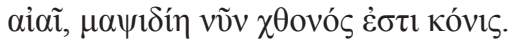

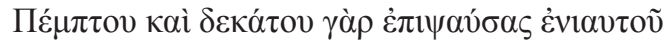

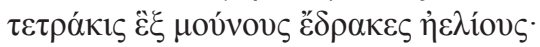

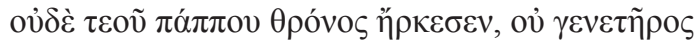

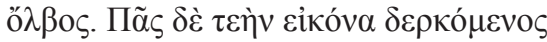

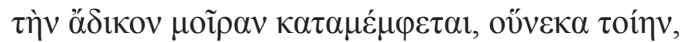

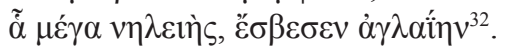

Como vemos, Verzosa ha escogido como principales modelos dos epigramas que están dedicados a muertes prematuras, como fue, en cierta medida, la de Rescius ${ }^{33}$. Pero, aparte de estos modelos que afectan a versos completos, otras expresiones recuerdan también a poemas del libro séptimo de la Anthologia Graeca. Por ejemplo,

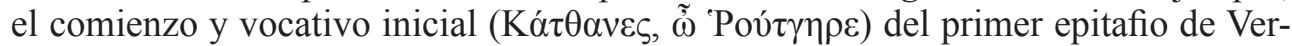
zosa puede tener que ver con un dístico del consular Juliano (considerado también prefecto de Egipto: A.P. 7.576$)^{34}$ :

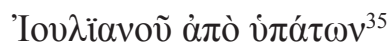

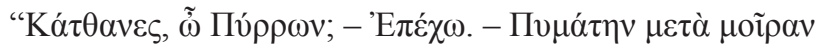

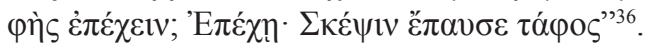

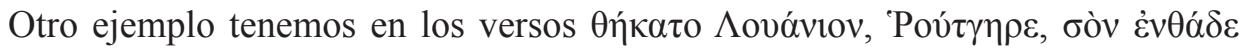

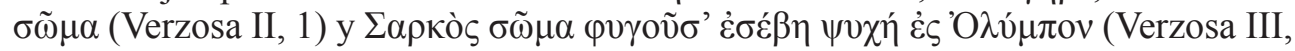

30 Sigo a Waltz (1960: 5, 109). El epigrama apareció en Láscaris (1494: f. 302). Puede verse también en Dübner (1871-1890: 1, 389, 489); Stadtmüller (1899: 410-412); Paton (1970: 322-323).

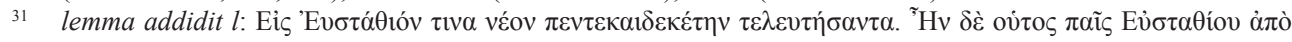

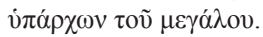

32 Traducción de los tres últimos versos: "Todo el que mire tu imagen culpará a la injusta Moira por apagar, ¡oh, gran impía!, tamaña gloria”.

33 Aunque por la expectativa de vida de la época los cincuenta años abrían la época de la vejez, los cuarenta y tantos años de Rescius (no se conoce la fecha de nacimiento, aunque se sitúa en torno a 1497) pueden considerarse prematuros para morir, sobre todo si se tienen en cuenta los frutos intelectuales que aquel profesor habría podido dar todavía para el mundo culto y, en especial, para sus discípulos.

34 Sigo a Waltz (1960, 5: 99). Véase también Lascaris (1494: 340-341); Dübner (1871-1890: I, 384, 487-488); Stadtmüller (1899: 396); Paton (1970: 308-309).

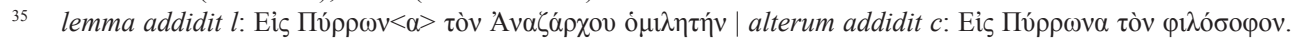
A $\mu$ oı $\beta$ ĩov.

36 Traducción: “¿Estás muerto, Pirro? —Lo dudo. —Después de tu último día, ¿dices que lo dudas? Lo dudas: esta tumba resuelve tu duda". El epigrama es una crítica a la escéptica actitud 'pirronista'. 
3). Ambos han podido tomar como modelo a A.P. 7.362, de Filipo de Tesalónica para el rétor $\mathrm{Aecio}^{37}$ :

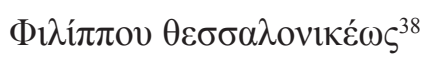

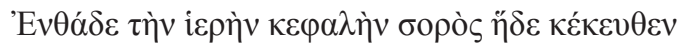

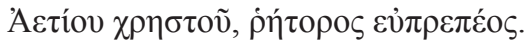

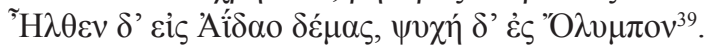

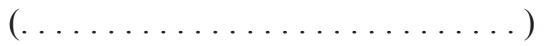

$(\ldots \ldots \ldots \ldots \ldots \ldots \ldots \ldots \ldots) \dot{\alpha} \theta \dot{\alpha} v \alpha \tau$. $\delta \ldots$

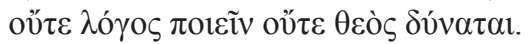

Este poema tiene un difícil problema textual precisamente en los versos posteriores a 'O $\lambda \nu \mu \pi \mathrm{ov}$. La tradición planudea aporta el siguiente pentámetro tras 'О $\lambda \nu \mu \pi \mathrm{ov}$ :

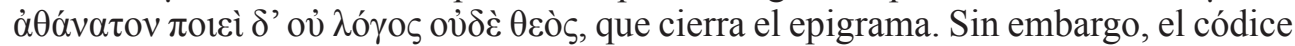
palatino tiene la lectura $\dot{\varepsilon} v$ 'O $\lambda \hat{\mu} \mu \pi \omega$ y continúa el verso siguiente así: $\tau \dot{\varepsilon} \rho \pi \varepsilon \theta^{\prime}$ ' $\alpha{ }^{\prime} \alpha$

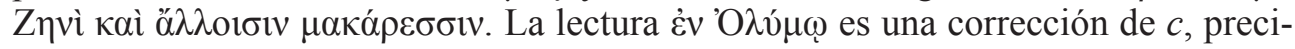
samente para unir este sintagma con el verso siguiente ( $\tau \dot{\varepsilon} \rho \pi \varepsilon \theta^{\prime}$ ŏ $\left.\mu \alpha . ..\right)$ y desligarlo del verbo ह̌ $\chi \chi \mu \mu \alpha$. Por su parte, en el códice palatino aparecía además, después de

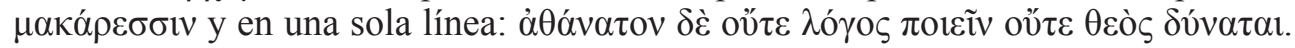

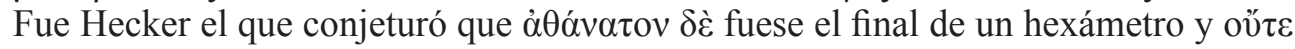

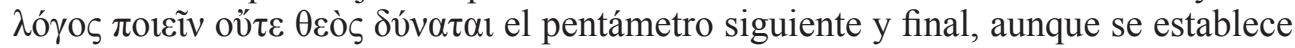

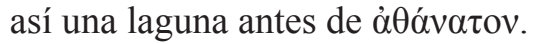

Por último, la expresión $\dot{\varepsilon} \theta \theta \dot{\delta} \delta \varepsilon \theta \eta \dot{\kappa} \alpha \tau$ (o similares) es muy frecuente en epitafios. En las notas de loci similes con que he acompañado el texto griego pueden verse otras dos coincidencias léxicas.

Y además de esto, si atendemos al tercero de los epigramas (en particular III 4-6), vemos que todo él gira en torno al juego de palabras entre $\sigma \tilde{\omega} \mu \alpha$ y $\sigma \tilde{\eta} \mu \alpha$ que usó Platón en sus tres famosos pasajes (Grg. 493a, Cra. 400c, Phdr. 250c) y que está presente en el platonismo desde entonces ${ }^{40}$.

\subsection{Una aportación a la historia de la Anthologia Graeca, deducida de los epigramas de Verzosa}

A la información dada arriba sobre la Anthologia Graeca debe añadirse que estudios recientes están confirmando las ideas expuestas por Cameron ${ }^{41}$. En primer lugar, que hay motivos convincentes para pensar que Erasmo adquirió o copió en Siena (1508) el códice que contenía la colección llamada después Palatina ${ }^{42}$. En segundo lugar, que Erasmo debió de dar a conocer el manuscrito a Tomás Moro al visitarlo en

37 Sigo a Waltz (1960: 4, 206-207). El texto está presente en Lascaris (1494: 332); Dübner (1871-1890: I, 341, 458-459); Stadtmüller (1899: 241-242); Paton (1970: 192-193).

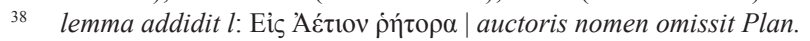

39 Traducción de los tres primeros versos: "Esta urna guarda aquí la sagrada cabeza del buen Aecio, el famoso orador. Su cuerpo se marchó al Hades, su alma al Olimpo".

40 Es amplísima la bibliografía sobre esta concepción del cuerpo y el alma. Véanse algunos hitos, por orden cronológico: Courcelle (1966); de Vogel (1986: 233-248); Bernabé (1995: 204-237).

41 Véase Cameron (1978 y 1993: 97-159); Van Miert (2011: 242).

$42 \quad$ Vid. Mcdonald (2013: 264). 
Londres el año siguiente. Por eso Moro, al publicar unos Progymnasmata en 1518, incluyó en ellos (era la última pieza antologada, el epigrama 18) un poema ausente de la colección planudea y solo presente en el que luego se llamaría Códice Palatino ( $P 23$ de la Biblioteca de Heidelberg) ${ }^{43}$.

Los Clement fueron una familia aristocrática católica que huyeron de Inglaterra al subir al trono Eduardo VI (1547). John Clement, discípulo y luego yerno de Moro, fue quien llevó entonces el códice a su casa de Lovaina ${ }^{44}$. Allí lo consultó en 1551 Henri Estienne, que luego publicó varios textos incluidos en aquel códice ${ }^{45}$, así como Gerard Falkenburg en 1566 (o poco después), que dejó constancia escrita de su consulta en casa de los Clement ${ }^{46}$.

A la vista de este periplo del códice que luego sería catalogado en Heidelberg con la sigla $P 23$, cobra relevancia la lectura ह̌ $\pi \lambda \varepsilon o$ del primer epigrama de Verzosa $(I, 7)$. Como sabemos, el final del epigrama casi calca el de otro de Paulo Silenciario (A.P. 7.560 ), que en la tradición planudea tenía la lectura é $\pi \lambda \varepsilon \tau$. Rescius y Verzosa, coincidentes en Lovaina entre 1542 y 1545, solo pudieron conocer, en versión impresa, la Anthologia de Planudes ${ }^{47}$. El epigrama de Silenciario fue editado por primera vez por Lascaris (1494: 300-301, con una segunda versión en páginas 305-306). Las dos versiones tenían la lectura ह̌ $\pi \lambda \varepsilon \tau$. Además, las ediciones impresas coetáneas editaron también $\ddot{\varepsilon} \pi \lambda \varepsilon \tau \mathrm{o}^{48}$. Es cierto que la lectura Ë $\pi \lambda \varepsilon o$ aparecía en los códices planudeos más tardíos (menospreciados en el paso de la colección a la imprenta) ${ }^{49}$, pero nada relaciona a esos códices con Lovaina.

Téngase en cuenta que la diferencia entre una lectura y otra es considerable ${ }^{50}$ : en la tradición planudea el autor se queja en términos generales (en tercera persona) de la injusticia de la Moira; en la palatina el autor se dirige directamente a la Moira para increparla. Parece claro que la elección de la segunda posibilidad en Verzosa se debió a conocer el Códice Palatino, antes incluso de que los Clement lo llevaran a Lovaina (1547). Es una hipótesis muy plausible que el propio Erasmo, durante su estancia en Lovaina, llevase allí el códice y lo compartiera con Rescius, a quien tanto ayudó; y de manos de Rescius pudo llegar fácilmente a Verzosa.

Vuelvo ahora a la pregunta sobre la que di una hipótesis al inicio: ¿cómo llegaron a Vulcanius los epigramas de Verzosa? Ciertamente no hay documentación para

$43 \quad$ Vid. Mcdonald (2013: 259).

44 Que habría adquirido de manos de Moro (Mcdonald 2013: 265); o que habría traído de su propia estancia italiana de 1522-1523, hipótesis esta última de Valentin Rose, citada en Waltz (1960: 1, XXXVIII).

45 Los Anacreontea, en París, 1554; los Idilios de Teócrito, Mosco y Bión, en Venecia, 1555; y seis epigramasadivinanzas añadidos a su edición de la Antología Planudea de 1566 (ausentes hasta entonces de la misma). Vid. Mcdonald (2013: 265).

46 Vid. Mcdonald (2013: 266).

47 En una de sus primeras ediciones: Venecia: Lascaris, (1494); Venecia: Aldo, 1503; Florencia, Giunti: 1519 y Aldo: 1521; y París: Badius Ascensius, 1531.

48 Véase Florilegium (1503: 301-305); Florilegium (1519: 258v, 260v); Florilegium (1521: 112-113); Florilegium (1531: 170).

49 Los agrupados en la llamada Sylloge Euphemiana, que acoge cuatro manuscritos de los siglos XV y xvi; y los de la llamada Sylloge $\Sigma^{\pi}$, que acabó encuadernada con P 23. Véase Waltz (1960: 1, LII-LIII); Mcdonald (2013: 263).

50 A diferencia de la lectura $\dot{\varepsilon} \varsigma$ 'O $\mathrm{O} \nu \mu \mu \pi \mathrm{ov}$ (en Verz. III, 3), coincidente con A.P. 7.362.3, donde la tradición palatina llevaba una corrección de $c$ en favor del complemento $\dot{v} v$ 'O $\lambda \hat{\nu} \mu \pi \omega$, que encajaría gramaticalmente con el resto de su versión, sin duda corrupta. Creo que esta coincidencia es mucho menos significativa que la anterior porque se trata del calco de un simple sintagma (usual, por otra parte) y no de versos enteros, así como por el estado problemático en que $P 23$ transmitía el texto. Lo único que esto podría apoyar es que Verzosa conociera también alguna de las ediciones hechas a partir de Planudes. 
responder con certeza. Pudo ser, como dije, en la primera estancia de Vulcanius en Lovaina, como estudiante (1555-1557); pero, no obstante, es bueno considerar que Vulcanius no fue ajeno a los viajes que hizo el Códice Palatino. Los Clement tuvieron también casa ( $\mathrm{y}$ biblioteca) en Malinas y, a pesar del saqueo español de 1572 , luego volvieron a tenerla y el códice estuvo allí hasta $1580^{51}$. Vulcanius tuvo alguna noticia del contenido de esa biblioteca, puesto que pidió a Thomas Clement (sin éxito) una copia de Cirilo de Alejandría para colacionar su manuscrito. Cuando Malinas sufrió otro saqueo por las tropas de Guillermo de Orange (1580), los libros y manuscritos de los Clement se dispersaron definitivamente y fueron vendidos por lotes al mejor postor. Uno de los compradores, según la fuente citada en nota anterior, fue Vulcanius. Si los epigramas de Verzosa se conservaron en el culto entorno lovaniense de los Clement (tanto en Lovaina como en Malinas), esa habría sido una vía para que los epigramas de Verzosa llegasen a Vulcanius y a la Biblioteca de la Universidad de Leiden ${ }^{52}$.

\subsection{Morfología, métrica y estilo de los epigramas}

Los rasgos morfológicos de los epigramas de Verzosa corresponden al dialecto jónico $^{53}$ y a la épica homérica; los métricos al hexámetro de Homero ${ }^{54}$. En cuanto a este segundo punto, Verzosa sigue la norma de la Antigüedad. En las cesuras de los hexámetros predomina la pentemímera, salvo dos versos (I 1 y III 1) que tienen, en cambio, la femenina del tercer pie, a la vez que la conjunción de trihemímera y heptemímera. Lo importante para este trabajo es que las cesuras de los epigramas de Verzosa no contradicen lo habitual en la versificación griega antigua ${ }^{55}$. No obstante, los versos II 1 y III 5, muestran una pausa tras el cuarto troqueo, lo que deshace el

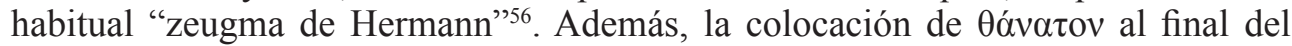
primer hemíepes del pentámetro (en I 2) muestra impericia, en cuanto que la vocal larga suele serlo por naturaleza y no, como aquí, por posición.

De todas formas, no deja de llamar la atención el dominio que tiene el joven Verzosa de la lengua griega y su profundo conocimiento de la Anthologia. Este hecho también apoya el que fuera docente y, quizás, su nombramiento como professor tras la muerte de Rescius.

Hemos visto que Verzosa toma varios epigramas funerarios como modelos: el 7.560 de Paulo Silenciario para Leoncio; el 7.602 de Agatías Escolástico para Eustacio, un joven de clase alta; el 7.576 de Juliano el prefecto de Egipto para el orador Pirro (o Pirrón); y el 7.362 de Filipo de Tesalónica para otro orador, Aecio. El primer

51 Véase, también para lo que sigue, Mcdonald (2013: 267-268).

52 Téngase en cuenta que no es impensable que Verzosa llegara a conocer a los Clement, puesto que estuvo en la corte de María Tudor y Felipe II en Londres, adonde volvieron los Clement para vivir allí mientras duró ese reinado, aunque con Isabel I retornaron definitivamente a Flandes.

53 Esto es algo que se compagina bien con que sus principales modelos estén en la Anthologia Graeca. Para lo referente al predominio del dialecto jónico en la colección, véase Gow \& Page (1965-1968: 1, xlv).

54 Como estudios métricos de carácter genérico y referidos a la Antigüedad, he consultado, Silva (1998: 207-223), Guzmán Guerra (1997) y West (1982 y 1987). Sobre la correptio épica, que está presente en el texto, véase en particular Guzmán Guerra (1997: 53) y Silva (1998: 207, n. 111); de igual manera, sobre la llamada versteckte Position ('posición enmascarada') véase Guzmán Guerra (1997: 50-51).

55 Sobre la naturaleza y disposición de las cesuras, como se sabe, hay diferentes opiniones, que aquí no interesa distinguir. Puede verse, no obstante, Guzmán Guerra (1997: 54-55); Silva (1998: 335-360), con bibliografía.

56 Véase sobre este zeugma Guzmán Guerra (1997: 56-57); Hermann (1816); Silva (1998, 483-486). 
y tercer epigrama de Verzosa tienen tres dísticos; el segundo tiene dos. Pues bien, Verzosa ha ido a buscar cauce para su inspiración en esas fuentes antiguas, pero no las ha seguido como un espejo. Así, su primer epigrama comienza con un vocativo que puede relacionarse con el inicio del epigrama de Juliano, pero el resto del primer dístico aporta de su propia cuenta una alabanza del conocimiento de Rescius sobre las lenguas griega y latina. El segundo dístico (I, 3-4) toca una idea que no está en las piezas de la Anthologia que han dejado huella léxica, pero que es un tópico del género: el fallecido es el afortunado, por haber alcanzado el Olimpo, mientras que el dolor y las lágrimas quedan para quienes le sobreviven ${ }^{57}$. El primer epigrama verzosiano acaba (es su tercer dístico) con la queja contra la Moira, esta vez sí, casi con las mismas palabras que Paulo Silenciario. Pero, aun así, Verzosa innova en el último verso, en el sentido de dar una mayor viveza a la expresión. Al uso de la segunda persona del verso penúltimo ( $\varepsilon \pi \lambda \varepsilon$ o Moĩ $\alpha$ ), él añade otra forma verbal igual

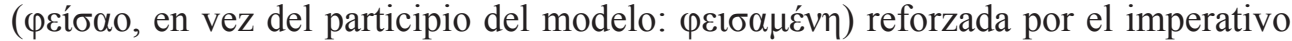
del principio de verso. También cambia el complemento de $\varphi \varepsilon i ́ \delta o \mu \alpha$ : pasa de ser la juventud a la piedad.

El segundo epigrama de Verzosa alude en su primer verso a la muerte de Rescius lejos de su patria y en el siguiente al dolor con el que ha quedado Verzosa. La muerte en tierra extranjera está en relación con la muerte de Leoncio, del que Silenciario dijo que no pudo contar ni siquiera con la presencia de sus padres. El segundo dístico del segundo epigrama de Verzosa es otra queja contra las Moiras y está tomada, con préstamos léxicos incluidos, del final del epigrama de Agatías Escolástico para Eustacio.

El tercer epigrama de Verzosa, después de dedicar, como acabamos de decir, el primer dístico a la bienaventuranza del difunto, trata en el segundo del conocido binomio $\sigma \tilde{\omega} \mu \alpha$ / $\sigma \tilde{\eta} \mu \alpha$ (con su insistencia en los ictus del segundo hemíepes: $\sigma \eta ́ \mu \alpha \tau 1$ $\sigma \tilde{\omega} \mu \alpha \tau \varepsilon o ̀ v)$; un binomio este que, aparte de estar en los pasajes ya señalados de Platón, aparece en el epigrama de Filipo a Aecio.

Verzosa sigue el método compositivo propio de los poetas humanistas; no obstante, me parece que Verzosa muestra aciertos de verdadero poeta (como ocurre en su producción neo-latina). Cuida el sonido especialmente. El primer epigrama es quizás el más conmovedor y basa su fuerza en diversos recursos principalmente fónicos. Los dos primeros dísticos acaban con palabras de parecido sonido: $\dot{\varepsilon} \xi \dot{\varepsilon} \varphi v \gamma \varepsilon \varsigma$, $\dot{\varepsilon} \xi \dot{\varepsilon} \lambda \iota \pi \varepsilon \varsigma$ (vocales breves átonas, de timbre parecido, entre las épsilon); y el tercero con otra que hace de contrapunto ( $\varepsilon v j \sigma \varepsilon \beta i ́ n s$, con iota acentuada entre dos vocales del mismo timbre).

Todos los hexámetros tienen cesura pentemímera, salvo el primero, que hace re-

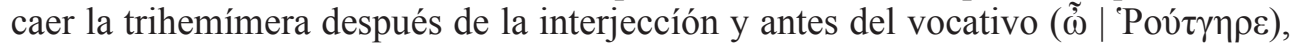
destacando así la expresión emotiva. De manera parecida, realza el adverbio $\kappa \rho \alpha \tau \varepsilon \rho \tilde{\omega} \varsigma(\mathrm{I}, 5)$, al situar tras él la cesura pentemímera y hacer recaer el tercer ictus en la última de sus sílabas. Lo mismo puede decirse del adverbio $\tau \alpha \chi \varepsilon ́ \omega \varsigma$ (II, 4) que acaba el primer hemíepes del pentámetro. Este mismo recurso puede verse en $\sigma o \tilde{v}$ (II, 2). Además, la oposición entre $\sigma \tilde{\omega} \mu \alpha$ y $\sigma \tilde{\eta} \mu \alpha$ en el tercer epigrama se apoya también en la aliteración de la silbante, clara sobre todo en $\Sigma \alpha \rho \kappa o ̀ \varsigma ~ \sigma \tilde{\omega} \mu \alpha$ $\varphi v \gamma о \tilde{v} \sigma$ '

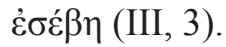

57 Por otro lado, esta misma idea sirve a Verzosa para el primer dístico de su tercer epigrama. 
Igualmente Verzosa cuida la disposición de las palabras. El verso II, 1 es un ejemplo especial de equilibrio, con el sujeto y verbo en la primera parte, el complemento en la segunda, y el centro reservado para el vocativo con el nombre de Rutgerus, lo mismo que $\sigma o \tilde{v}$ en el siguiente. Son significativas las palabras situadas en los

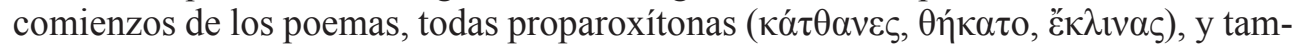

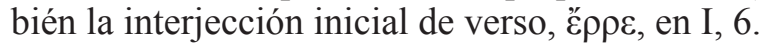

Creo, en definitiva, que Verzosa consiguió, dentro de lo convencional que hay siempre en la literatura (y más de lenguas meramente literarias), transmitir su emoción por la muerte de alguien a quien debió no poco durante su estancia en Lovaina.

\section{Conclusiones}

El descubrimiento de tres epigramas griegos escritos en Lovaina por el humanista Juan de Verzosa, dedicados a lamentar la muerte de Rutgerus Rescius en 1545, contribuye a avalar la hipótesis de que el español inició una carrera docente en la Universidad de Lovaina en relación con el profesor flamenco. Asimismo, los epigramas suponen una aportación a la historia del itinerario que hizo el Códice Palatino (antes de que fuera 'descubierto' por Sumaise en Heidelber en 1606) y, por tanto, a la historia también de la Anthologia Graeca.

\section{Bibliografía}

A) Fuentes primarias de información manuscritas:

Library of the University of Leiden, Vulcanius collection: ms. Vul. 103 I.

B) Fuentes primarias de información impresas:

Alphabetum Graecum (1536), Alphabetum Graecum una cum multis aliis, quae expeditum quem reddunt ad grammaticam Graecam capessendam, Louvain, Rutgerus Rescius voor Jan Cock.

Aristophanes (1537), Aves, Louvain, Rutgerus Rescius.

Chrysostomus, St Johannes (1529), Peri tou, oti pollou men axiōmatos, dyskolon de episkopein, dialogoi ex. [De sacerdotio sive quod magnae sit dignitatis, sed difficile, episcopum agere, dialogi duo], Louvain, Rutgerus Rescius \& Joannes Sturmius.

Clenardus, Nicolaus (1530), Institutiones in linguam Graecam, Louvain, ex officina Rutgerus Rescius \& Joannes Sturmius.

De Torres, Luis (1577), Ioannis Verzosae epistolarum libri IIII, Panhormi, Mayda.

De Verzosa, Juan (1544), De prosodiis liber absolutissimus, nunc primum in gratiam liguae Graecae studiosorum editus, Leuven, Rutgerus Rescius.

Euripides (1537), Andromachē, Louvain, Rutgerus Rescius.

Evangelia (1541), Evangelia Graeca e Novo Testamento excerpta, quae domincis, et aliis festis diebus leguntur in templis, Louvain, in officina Rutgerus Rescius.

Florilegium diuersorum epigrammatum (1503), Venecia, Aldo Manuzio. He usado el ejemplar digitalizado (en formato pdf) del Münchener Digitalizierung Zentrum de la Bayerischen Staatsbibliothek localizable en internet: https://reader.digitale-sammlungen.de//de/fs1/object/display/ bsb11263333_00005.html (disponible a 15 de agosto de 2020). Como los folios del volumen no están numerados, me he visto obligado a citar por los fotogramas de esta reproducción. 
Florilegium diuersorum epigrammatum (1519), Florencia, Giunti.

Florilegium diuersorum epigrammatum (1521), Florencia, Aldo Manuzio.

Florilegium diuersorum epigrammatum (1531), Paris, Ascensius.

Hesiodus (1538), Erga kai èmerai, Louvain, Rutgerus Rescius.

Hippocrates (1533), Aphorismi, ex diversorum codicum collatione, Louvain, ex officina Rutgerus Rescius ven. Bartholomaeus Gravius.

Homerus (1535), Ulyssea, Batrachomyomachia, hymnoi 32, Louvain, ex officina Rutgerus Rescius voor Bartholomaeus Gravius.

Homerus (1532), Ilias. Iliados 1, Louvain, Rutgerus Rescius voor Bartholomaeus Gravius.

Lascaris, Janus (1494), Anthologia diuersorum epigrammatum, [Florencia: Lorenzo de Alopa]. He usado para acceder a Lascaris el ejemplar digitalizado (en formato pdf) del Münchener Digitalizierung Zentrum de la Bayerischen Staatsbibliothek, disponible en internet a 15 de agosto de 2020: http://daten.digitale-sammlungen.de/ db/0006/bsb00065290/ images/index.html?seite $=00001 \& l=e n$. Como los folios del volumen no están numerados, cito por los fotogramas de esta reproducción. Es el único caso de esta forma de cita, además del Florilegium de 1503.

Plato (1529), Sōkratous apologia. [Socratis apologia], Louvain, Rutgerus Rescius \& Joannes Sturmius.

Plato (1529a), Kritōn è peri praktou. [Criton], Louvain, Rutgerus Rescius \& Joannes Sturmius.

Xenophon (1530), Symposion, Louvain, Rutgerus Rescius \& Joannes Sturmius.

C) Fuentes secundarias de información:

Bernabé, Alberto (1995), «Una etimología platónica: $\Sigma \Omega M A-\Sigma H M A »$, Philologus 139.2: 204-237.

BibliothèqueNationaleFrançaise(ed.),ActaRectoriaParisiensisUniversitatis,7vols.(Latin99539957), disponible en internet en URL (consultada en 1/3/2020): https://gallica.bnf.fr/services/ engine $/$ search $/$ sru?operation $=$ searchRetrieve\&version $=1.2 \&$ query $=\% 28 \mathrm{dc}$.title $\% 20$ all $\% 20$ $\% 22$ Acta $\% 20$ rectoria $\% 20$ universitatis $\% 20$ Parisiensis $\% 22 \% 29 \&$ keywords $=$ Acta $\% 20$ rectoria\%20universitatis\%20Parisiensis\&suggest $=1$.

Brockliss, Lawrence W. B. (1976), The University of Paris in the Sixteenth and Seventeenth Centuries, Cambridge, University of Cambridge.

Cameron, Alan (1987), «Sir Thomas More and the Greek Anthology», en Karl-Ludwig Selig \& Robert Somerville (eds.), Florilegium Colombianum: Essays in Honor of Paul Oskar Kristeller, New York, Italica Press: 187-198.

Cameron, Alan (1993), The Greek Anthology: from Meleager to Planudes, Oxford, Oxford University Press.

Cazes, Hélène (ed.) (2010), Bonaventura Vulcanius, Works and Networks. (Bruges 1538 Leiden 1614), Leiden - Boston, Brill.

Courcelle, Pierre (1966), «Le corps-tombeau (Plato, Gorgias 493a, Cratyle 400c, Phèdre 250c)», REA 68: 101-112.

De Vocht, Henri (1927), Inventaire des archives de l'Université de Louvain (1526-1797) aux archives générales du royaume à Bruxelles, Louvain, Librairie universitaire, Ch. Uystpruyst.

De Vocht, Henri (1934), Texts and Studies about Louvain Humanists in the First Half of the $X V I^{\text {th }}$ Century: Erasmus, Vives, Dorpius, Clenardus, Goes, Moringus, Louvain, Librairie universitaire, Ch. Uystpruyst.

De Vocht, Henri (1951-1955), History of the Foundation and the Rise of the Collegium Trilingue Lovaniense, 1517-1550, 4 vols. Louvain, Librairie universitaire. 
Del Pino, Eduardo (2003), «Grudia me tenuit cultrix studiosa Mineruae: textos relacionados con con la estancia en Lovaina del humanista español Juan de Verzosa», $C F C(L)$ 23.1: 171-209.

Del Pino, Eduardo (2006), Juan de Verzosa. Epistolas, Madrid - Alcañiz: Consejo Superior de Investigaciones Científicas - Instituto de Estudios Humanísticos.

Del Pino, Eduardo (2016), «En la estela de Horacio: una epístola latina inédita de Giacinto Frangipane», Euphrosyne 44: 153-171.

Del Pino, Eduardo (2017a), «Un epicedio latino de Girolamo Fracastoro falsamente atribuido al escritor español neolatino Juan de Verzosa», NLJ 19: 339-367.

Del Pino, Eduardo (2017b), «La versión latina de Bonaventura Vulcanius de las 'Coplas a la muerte de su padre’ del español Jorge Manrique», Bibl.H\&R 79. 2: 395-417.

Del Pino, Eduardo (2019), «La 'Biblia Graeca' de Bonaventura Vulcanius. Nuevos datos del proyecto a partir de sus cartas y poemas latinos»), Latomus 78.4: 936-971 (doi: 10.2143/ LAT.78.4.3287638).

De Vogel, Cornelia Johanna (1986), Rethinking Plato and Platonism, Leiden, Brill.

De Vries de Heekelingen, Herman (1923), Correspondance de Bonaventura Vulcanius pendant son séjour à Cologne, Genève et Bâle (1573-1577): précédée de quelques lettres écrites avant cette époque, Den Haag, Nijhoff.

Dewitte, Alfons (1983), «Vulcanius Brugensis. Hoogleraarambt, correspondenten, edita», Sacris erudiri 26: 311-362.

Dübner, K. Frederic (1871-1890), Epigrammatum Anthologia Palatina cum Planudeis et appendice noua epigrammatum ex libris et marmoribus ductorum, annotatione inedita Boissonnadii, Chardonis de la Rochette, Bothii, partim inedita Iacobsii, metrica uersione Hugonis Grotii, apparatu critico et breuibus comentariis, Paris, Ambrosio Firmin-Didot.

Deutscher, Thomas B. \& Erbe, M. (2003), «Rutgerus Rescius», en Pieter G. Bietenholz \& Thomas Brian Deutscher (eds.), Contemporaries of Erasmus: a biographical register of the Renaissance and Reformation, 3 vols, Toronto - Buffalo - London, The University of Toronto Press: 3, 142-144.

Farge, James K. (2006), Students and teachers at the University of Paris: the generation of 1500: a critical edition of Bibliothèque de l'Université de Paris (Sorbonne) Archives, Registres 89 and 90, Leiden - Boston, Brill.

García Pinilla, Ignacio J. (2020), «Bonaventura Vulcanius en España: algunos poemas», en Florian Schaffenrath \& María Teresa Santamaría Hernández (eds.), Acta Conventus NeoLatini Albasitensis: Proceedings of the Seventeenth International Congress of Neo-Latin Studies (Albacete 2018), Leiden, Brill: 259-272 (doi:10.1163/9789004427105_019).

Gow, Andrew Farrar \& Page, Denys Lionel (1965-1968), The Greek Anthology. Hellenistic Epigrams, 2 vols. Cambridge, Cambridge University Press.

Gow, Andrew Farrar \& Page, Denys Lionel (1968), The Greek Anthology. The Garland of Philip, Cambridge, Cambridge University Press.

Guzmán Guerra, Antonio (1997), Manual de métrica griega, Madrid, Ediciones Clásicas.

Hermann, Gottfried (1816), Elementa Doctrinae Metricae, Leipzig, apud Gerh. Fleischerum Iuniorem.

Jacobs, Friedrich (1794-1814), Anthologia Graeca, Leipzig, Dyck.

Jacobs, Friedrich (1813-1817), Anthologia Graeca ad fidem codicis olim Palatini nunc Parisini, Leipzig, Dyck.

Janssens, Gustaaf (1992), «Españoles y Portugueses en los medios universitarios de Lovaina (siglos XV y XVI)», en Jan Lechner (ed.), Contactos entre los Países Bajos y el mundo ibérico, Amsterdam, Atlanta, Rodopi: 13-29. 
Maestre, José M. '(2002), Juan de Verzosa. Anales del reinado de Felipe II, Madrid - Alcañiz, Consejo Superior de Investigaciones Científicas - Instituto de Estudios Humanísticos.

Mcdonald, Bradley (2013), «Thomas More, John Clement and the Palatine Anthology», Bibl.H\&R 75: 259-270.

Montes Cala, Guillermo (1993), «La creación literaria en griego antiguo durante el Renacimiento: consideraciones metodológicas para su estudio a través de tres epigramas de Poliziano», en J. M. ${ }^{a}$ Maestre, L. Charlo \& J. Pascual (eds.), Humanismo y pervivencia del mundo clásico, Alcañiz, Instituto de Estudios Humanísticos: I, 1, 79-209.

Nèvo, F. (1856), «Mémoires historiques et littéraires sur le collége des Trois-Langues à l'université de Louvain», en Mémoires couronnés et mémoires des savants étrangers publiés par l'Académie royale des sciences, des lettres et des beaux-arts de Belgique 28: 202-207.

Papy, Jan (ed.) (2017a), Het Leuvense Collegium Trilingue 1517-1797: Erasmus, humanistische onderwijspraktijk en het nieuwe taleninstituut Latijn-Grieks-Hebreeuws, Leuven, Peeters.

Papy, Jan (2017b), Erasmus'droom: het Leuvense Collegium Trilingue 1517-1797, Leuven, Peeters.

Paton, W. R. (1979-1983) (=1916-1918), The Greek Anthology, The Loeb Classical Library, vol. $1(1980=1916)$, vol. $2(1970=1917)$, vol. 3 (1983=1917), vol. 4 (1979=1918), vol. 5 (1979=1918), Cambridge MA - London, Harvard University - William Heinemann LTD.

Pérez-Morillo, María del Mar (2002), Juan de Verzosa. Charina siue Amores, Madrid - Alcañiz, Consejo Superior de Investigaciones Científicas - Instituto de Estudios Humanísticos.

Pérez-Morillo, María del Mar (2009-2013), «Juan de Verzosa», Diccionario Biográfico Español, Real Academia de la Historia: 49, 783-786.

Reusens, Edmond Henri Joseph (1977), Documents relatifs à l'Histoire de l'Université de Louvain (1425-1797). Tables de Notices consacrées aux membres de l'Université, Catalogues, Inventaires et Répertoires de la Bibliothèque Centrale IV, Louvain, Université Catholique de Louvain.

Schillings, Arnold Hubert (1903-1967), Matricule de l'Université de Louvain (1426-1789), 10 vols., Brussel, Académie royale des sciences, des lettres et des beaux-arts de Belgique.

Silva, Tomás (1998), The hexameter of Oppian of Anazarbus and Oppian of Apamea, Cádiz, Universidad de Cádiz, publicado en microfichas. ISBN: 84-7786-632-5.

Silvano, Luigi (2014), «Per l'edizione della 'Disputa tra un ortodoso e un latinofrone sequace di Becco sulla processione dello Spirito Santo’ di Giorgio Moschampar. Con un inedito di Bonaventura Vulcanius», Medioevo greco 14: 229-265.

Stadtmüler, Hugo (1894-1906), Anthologia Graeca epigrammatum Palatina cum Planudea, 3 vols. (1894, vol. 1), (1899 vol. 2), (1906 vol. 3), Lipsiae, in aedibus B. G. Teubneri.

Stefani, Matteo (2019), «Bonaventura Vulcanius traduttore della Catena in Ioannem», HumLov 68.2: 317-359 (doi: https://doi.org/10.30986/2019.317).

Tournoy, Gilbert (2011a), «A Life-Long Dream: Bonaventura Vulcanius and His Edition of St. Cyril of Alexandria (I)», Calamus Renascens 12: 185-255.

Tournoy, Gilbert (2011b), «La correspondance de Bonaventura Vulcanius: quelques notes de lectura», HumLov 60: 315-325.

Van Dam, Harm-Jan (2010), “The honour of letters": Bonaventura Vulcanius, scholar and poet, en H. Cazes (ed.), Bonaventura Vulcanius, Works and Networks. (Bruges 1538 Leiden 1614), Leiden - Boston, Brill: 47-68.

Van Miert, Dirk (2011), «Joseph Scaliger, Claude Saumaise, Isaac Casaubon and the Discovery of the Palatine Anthology (1606)», JWI 74: 241-246. 
Waltz, Pierre (1960), Anthologie grecque, Tome 1 (Livres I-IV); Tome 4 (Livre VII, Épigr. 1-363); Tome 5 (Livre VII, Épigr. 364-748), Paris, Les Belles Lettres.

West, Martin Litchfield (1982), Greek Metre, Oxford, Oxford University Press.

West, Martin Litchfield (1987), Introduction to Greek Metre, Oxford, Oxford University Press.

\section{Ilustraciones}

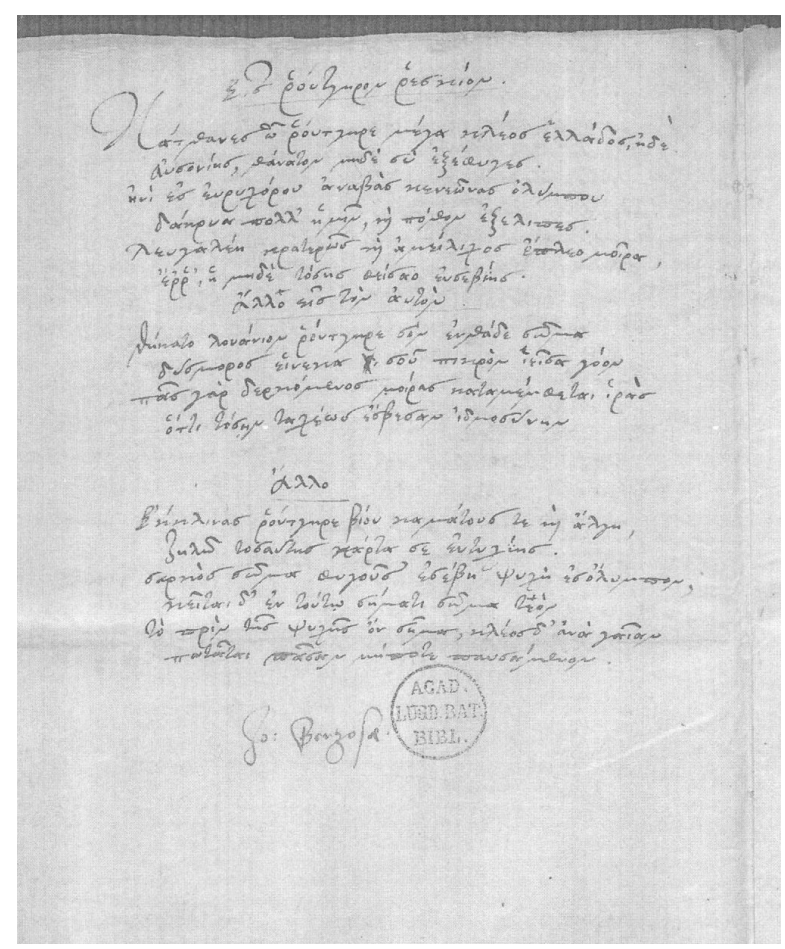

Ms. BUL Vul. 103 I, f. $2^{\mathrm{v}}$.

Reproducido por gentileza de la Bibliotheek Universiteit Leiden. 